\title{
Direct and Large-Eddy Simulation of Turbulent Flows on Composite Multi-Resolution Grids by the Lattice Boltzmann Method
}

\author{
Hatem Touil, ${ }^{1}$ Denis Ricot, ${ }^{2}$ and Emmanuel Lévêque ${ }^{1}$ \\ ${ }^{1}$ Laboratoire de Physique de l'École normale supérieure de Lyon, CNRS, Université de Lyon \\ ${ }^{2}$ Renault, Technocentre, 1 av. du Golf, F-78280 Guyancourt, France
}

(Dated: February 5, 2013) 


\begin{abstract}
In order to properly address the simulation of complex (weakly compressible) turbulent flows, the lattice Boltzmann method, originally designed for uniform structured grids, needs to be extended to composite multi-domain grids displaying various levels of spatial resolution. Therefore, physical conditions must be specified to determine the mapping of statistical information (about the populations of moving particles) at the interface between two domains of different resolutions. It is here argued that these conditions can express quite simply in terms of the probability distributions of the underlying discrete-velocity Boltzmann equation. Namely, the continuity of the mass density and fluid momentum is fulfilled by imposing the continuity of the equilibrium part of these distributions, whereas the discontinuity of the rate-of-strain tensor is ensured by applying a "spatial transformation" to the collision term of the discrete-velocity Boltzmann equation. This latter condition allows us to explicitly account for the subgrid-scale modeling in the treatment of resolution changes. Test computations of a turbulent plane-channel flow have been considered. The lattice Boltzmann scheme relies on the standard D3Q19 lattice in a cell-vertex representation, and uses the BGK approximation for the collision term. A shear-improved Smagorinsky viscosity is used for the subgrid-scale modeling. In a quasi-Direct Numerical Simulation at $\operatorname{Re}_{\tau}=180$ (with two levels of resolution) the results are found in excellent agreement with reference data obtained by a highresolution pseudo-spectral simulation. In a Large-Eddy Simulation at $\operatorname{Re}_{\tau}=395$ (with three levels of resolution) the results compare very well with high-resolution reference data. The accuracy is improved in comparison with a large-eddy simulation based on finite-volume discretization with the same subgrid-scale viscosity model and comparable grid resolution. This study demonstrates the good capabilities of the lattice Boltzmann method to handle both Direct and Large-Eddy Simulations of turbulent flows with grid resolutions comparable to those commonly used in simulations based on standard discretization methods, e.g. pseudo-spectral or finite-volume methods.
\end{abstract}

PACS numbers: 47.11.Qr, 47.11.-j, 47.27.E- 


\section{INTRODUCTION}

It is characteristic of fluid flows to develop finer and finer dynamical structures, e.g. shear layers or elongated vortices, as the Reynolds number increases [1]. Adapting the whole resolution of a numerical simulation to the size of these finest structures implies considerable computational efforts. So in practice, it is desirable to refine the grid only in regions where a finer resolution is needed, and use a coarser grid in the rest of the domain. This requirement becomes mandatory for the Large-Eddy Simulation (LES) of turbulent flows, in which grid refinement is often necessary near solid boundaries whereas a coarser resolution can be used in the bulk of the flow, on the condition to account for subgrid-scale turbulent dynamics [2]. Despite its relative novelty (see [3] for a seminal article), the lattice Boltzmann (LB) method has already proven its potential to simulate complex flows on (sufficiently fine) uniform grids [4]. However, improvements are still needed to tackle turbulent flows on composite multiresolution grids, the main difficulty arising from the "lack of deformability" of the lattice. As recently quoted by Sagaut [5], the development of LES within the framework of the lattice Boltzmann method is still at a very early stage.

While conventional methods start with a continuous description of the fluid at a macroscopic level, e.g. the Navier-Stokes equations, the LB method considers the fluid at a mesoscopic level, intermediate between the microscopic and the macroscopic [6]. Concretely, the fluid is modeled as populations of (fictitious) particles that collide, re-distribute and propagate along the different links of a discrete lattice, so that the desired "collective dynamics" can be recovered in the continuous limit [7]. The complexity of the flow emerges from the repeated application of simple rules of collision and streaming possibly supplemented by bounce-back or reconstruction procedures at the boundaries [8]. The LB method expresses the evolution of the probability distributions of these populations on the lattice, or grid. Originally, the method was designed for simple structured grid with a constant spacing in the three directions. Nevertheless, multi-resolution may be fulfilled by embedding domains of different (spatial) resolutions within the original grid (see Fig. 1). In that situation, some specific conditions are required to determine the mapping of the probability distributions at the boundary between two resolution domains. For the simulation of turbulent flows, this mapping is essential since it should account for the discontinuity of the rate of strain (related to the change of spatial resolution) and encompass effects related to possible subgrid-scale 


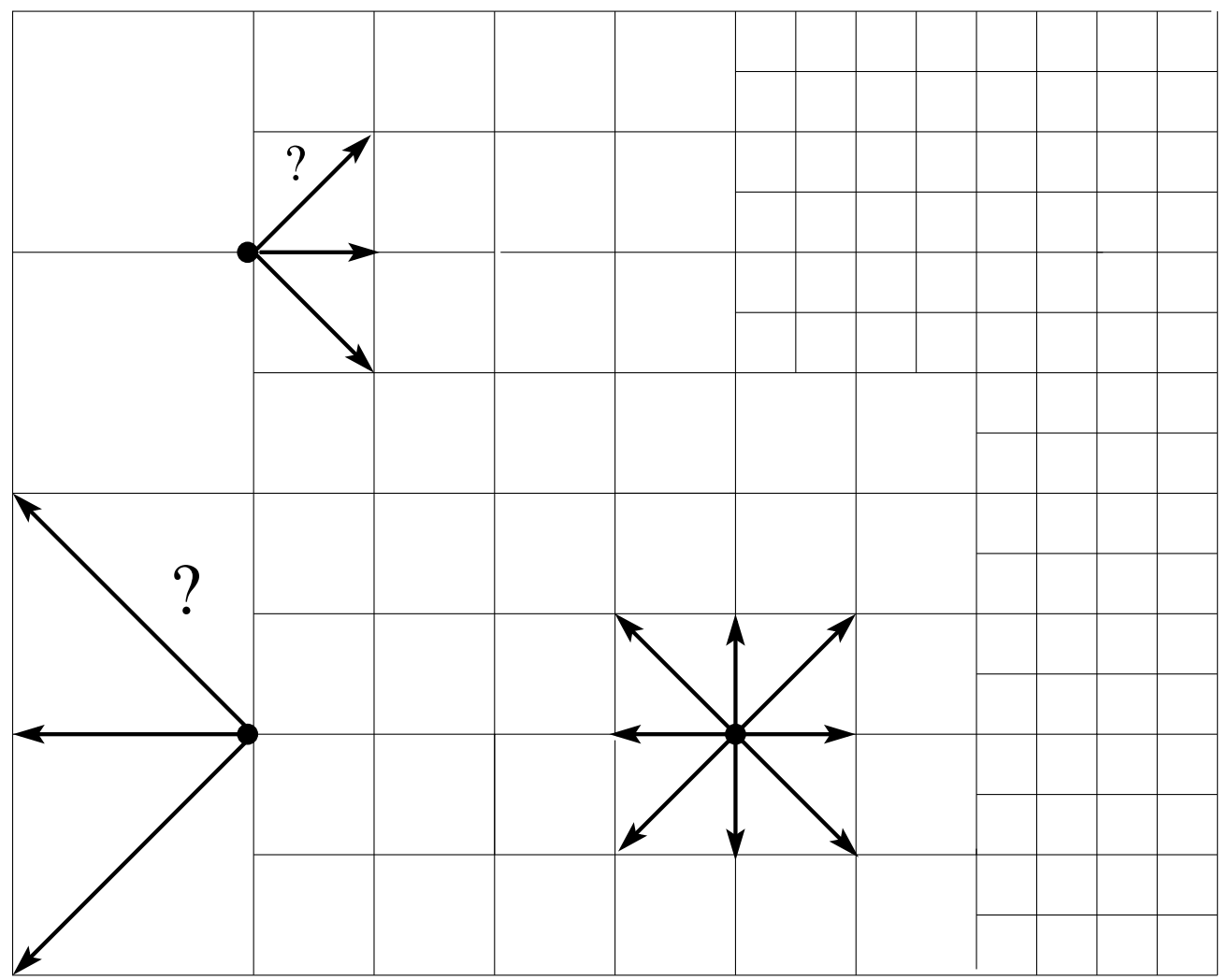

FIG. 1: Sketch of a (two-dimensional) multi-domain/multi-resolution grid in a cell-vertex representation. Inside each domain, particles can move towards neighboring nodes according to a discrete set of velocities. Some inconsistency in the connectivity of boundary nodes requires to suitably transmit the statistics of moving particles at the boundary of each domain.

turbulent dynamics.

In section II, the general framework of the LB method is briefly recalled and the position of the problem is presented. Our proposal is introduced and discussed with respect to existing works in section III. In section IV, the results from test computations of a turbulent channel flow are reported and compared with reference data from Direct and Large-Eddy Simulations. Finally, conclusions and perspectives are drawn in the last section. 


\section{THE GENERAL FRAMEWORK OF THE LB METHOD AND THE POSITION OF THE PROBLEM}

The LB method originates from the Boltzmann transport equation restricted to a finite set of velocities $\left\{\mathbf{c}_{\alpha}\right\}$ in phase-space:

$$
\frac{\partial f_{\alpha}(\mathbf{x}, t)}{\partial t}+c_{\alpha i} \frac{\partial f_{\alpha}(\mathbf{x}, t)}{\partial x_{i}}=-\frac{1}{\tau}\left(f_{\alpha}(\mathbf{x}, t)-f_{\alpha}^{\mathrm{eq}}(\mathbf{x}, t)\right)
$$

where $f_{\alpha}(\mathbf{x}, t) \mathrm{d}^{3} \mathbf{x}$ represents the probability (at time $t$ ) for a fictitious particle of fluid with the velocity $\mathbf{c}_{\alpha}$ to find itself within the elementary volume $\mathrm{d}^{3} \mathbf{x}$ about the position $\mathbf{x}$. Eq. (1) is usually called the discrete-velocity Boltzmann equation. It is essentially a balance of probability per unit volume, in which the BGK approximation $-\left(f_{\alpha}-f_{\alpha}^{\text {eq }}\right) / \tau$ is adopted in place of the complicated collision operator of the Boltzmann equation [9]; $\tau$ is a relaxation time related to the collision process. Indeed, it is thought that most details hidden in the collision operator play no role at the macroscopic level. It is therefore replaced with a much handier expression retaining only the basic features of fluid dynamics (at a mesoscopic level).

If the fluid has constant properties and its mass is conserved, $f_{\alpha}(\mathbf{x}, t)$ may also be viewed (provided a proper normalization) as the mass of fluid per unit volume that moves with the velocity $\mathbf{c}_{\alpha}$. The main strenght of the LB method is to prescribe the restricted sets of velocities, $\left\{\mathbf{c}_{\alpha}\right\}$, and equilibrium distributions, $\left\{f_{\alpha}^{\text {eq }}\right\}$, so as to recover (up to some order) the Navier-Stokes dynamics for the mass density and fluid momentum

$$
\begin{aligned}
& \rho(\mathbf{x}, t) \equiv \sum_{\alpha} f_{\alpha}(\mathbf{x}, t)=\sum_{\alpha} f_{\alpha}^{\mathrm{eq}}(\mathbf{x}, t) \\
& (\rho \mathbf{u})(\mathbf{x}, t) \equiv \sum_{\alpha} f_{\alpha}(\mathbf{x}, t) \mathbf{c}_{\alpha}=\sum_{\alpha} f_{\alpha}^{\mathrm{eq}}(\mathbf{x}, t) \mathbf{c}_{\alpha}
\end{aligned}
$$

by applying the Chapman-Enskog expansion method [7]. Therefore, a common practice is to consider the so-called D3Q19 lattice (in three dimensions) with 19 possible velocities [10], as sketched in Fig. 2. The (isothermal) equilibrium distribution expresses as

$$
f_{\alpha}^{\mathrm{eq}}(\rho, \mathbf{u})=\sum_{i j} w_{\alpha} \rho\left(1+\frac{u_{i} c_{\alpha i}}{c_{s}^{2}}+\frac{u_{i} u_{j} Q_{\alpha i j}}{2 c_{s}^{4}}\right) \quad \text { with } \quad Q_{\alpha_{i j}}=c_{\alpha i} c_{\alpha j}-\delta_{i j} c_{s}^{2} .
$$

The weighting coefficients are given by $w_{0}=1 / 3$ for the "center", $w_{1 \ldots 6}=1 / 18$ for the "faces" and $w_{7 \ldots 18}=1 / 36$ for the "edges". Finally, to ensure physical consistency, the constant velocity $c_{s}$ refers to the speed of sound (in the fluid) and the relaxation time $\tau$ is linked to the kinematic viscosity of the fluid by $\tau=\nu / c_{s}^{2}[11]$. 


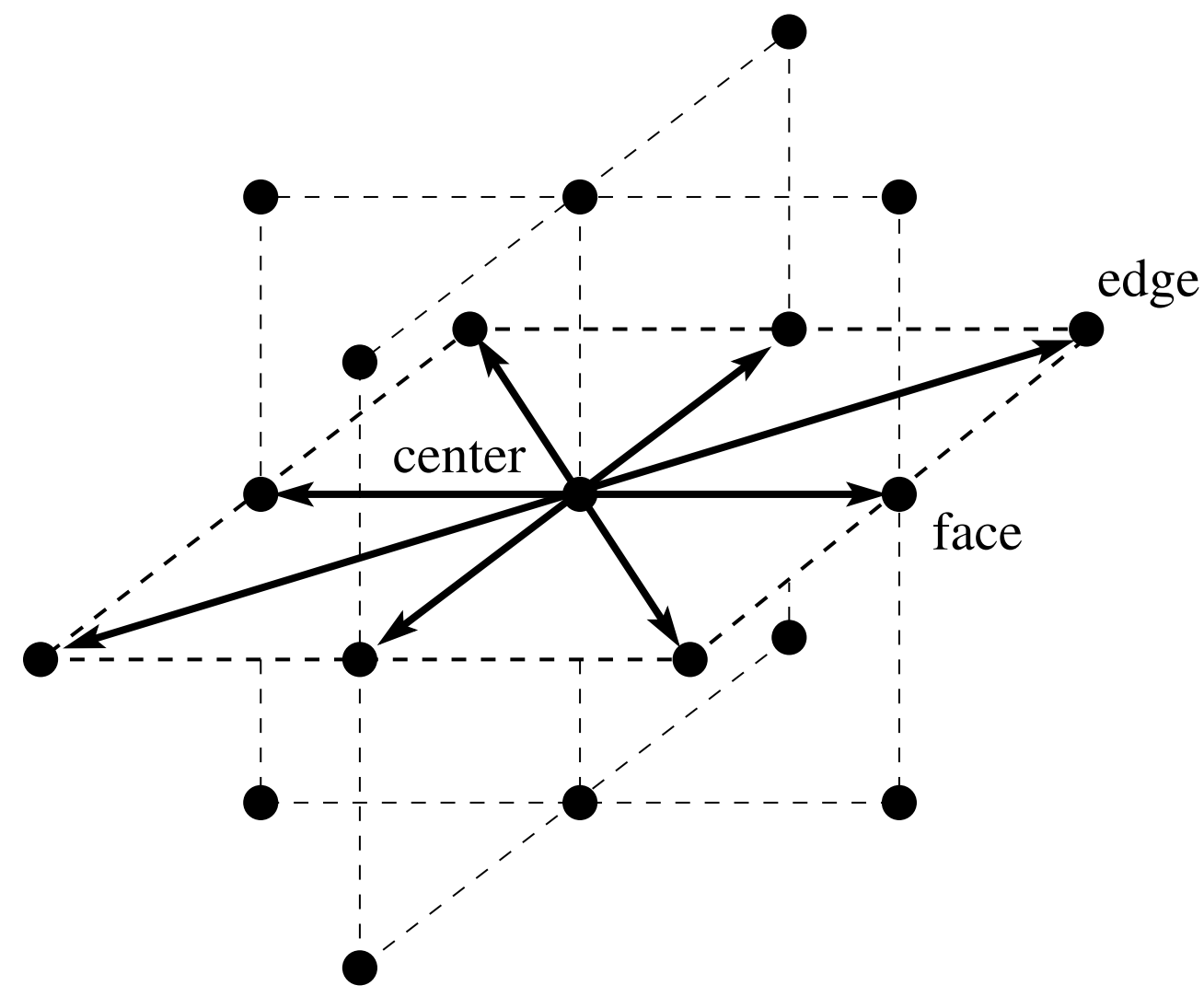

FIG. 2: The set of discrete velocities (including null velocity) of the D3Q19 lattice. For clarity, only the velocities in the horizontal plane are displayed. These 19 velocities can be grouped into three categories of vectors pointing respectively to the "center" (null vector), the "faces" and the "edges" of a cube. By construction, the respective velocities satisfy $\left|\mathbf{c}_{0}\right|^{2}=0,\left|\mathbf{c}_{1 \ldots 6}\right|^{2}=3 c_{s}^{2}$ and $\left|\mathbf{c}_{7 \ldots 18}\right|^{2}=6 c_{s}^{2}$.

In the LB scheme, the discretization of space stems directly from the discrete set of velocities so that each (fictitious) particle of fluid moves exactly to a neighboring lattice node during one timestep $\Delta t$. The integration of the discrete-velocity Boltzmann equation along these characteristics then yields

$$
\begin{array}{r}
f_{\alpha}\left(\mathbf{x}+\mathbf{c}_{\alpha} \Delta t, t+\Delta t\right)-f_{\alpha}(\mathbf{x}, t)=-\frac{1}{\tau} \int_{0}^{\Delta t}\left(f_{\alpha}\left(\mathbf{x}+\mathbf{c}_{\alpha} s, t+s\right)-f_{\alpha}^{\mathrm{eq}}\left(\mathbf{x}+\mathbf{c}_{\alpha} s, t+s\right)\right) d s \\
\approx-\frac{\Delta t}{2 \tau}\left(f_{\alpha}\left(\mathbf{x}+\mathbf{c}_{\alpha} \Delta t, t+\Delta t\right)-f_{\alpha}^{\mathrm{eq}}\left(\mathbf{x}+\mathbf{c}_{\alpha} \Delta t, t+\Delta t\right)+f_{\alpha}(\mathbf{x}, t)-f_{\alpha}^{\mathrm{eq}}(\mathbf{x}, t)\right)
\end{array}
$$

by approximating the integral by means of the (second-order) trapeziodal rule. If the "lattice-based" probability distribution

$$
g_{\alpha}(\mathbf{x}, t)=f_{\alpha}(\mathbf{x}, t)+\frac{\Delta t}{2 \tau}\left(f_{\alpha}(\mathbf{x}, t)-f_{\alpha}^{\mathrm{eq}}(\mathbf{x}, t)\right)
$$


is used instead of $f_{\alpha}(\mathbf{x}, t)$, the previous equation eventually simplifies as

$$
g_{\alpha}\left(\mathbf{x}+\mathbf{c}_{\alpha} \Delta t, t+\Delta t\right)=g_{\alpha}(\mathbf{x}, t)-\frac{\Delta t}{\tau_{g}}\left(g_{\alpha}(\mathbf{x}, t)-g_{\alpha}^{\mathrm{eq}}(\mathbf{x}, t)\right) \quad \text { with } \tau_{g}=\tau+\frac{\Delta t}{2} .
$$

This equation is usually considered as the fundamental equation of the LB scheme. The positions $\mathbf{x}$ are now restricted to the lattice nodes occupied by the particles at the discrete instants $t, t+\Delta t$, etc. In this regard, Eq. (5) may be truly interpreted as a (second-order) space-and-time discretized version of Eq. (1). However, the solution $g_{\alpha}$ is not exactly the genuine probability distribution of the discrete-velocity Boltzmann equation. It shares the same equilibrium part with $f_{\alpha}\left(g_{\alpha}^{\mathrm{eq}}=f_{\alpha}^{\mathrm{eq}}\right)$ but the non-equilibrium parts are different and related by

$$
g_{\alpha}^{\text {neq }} \equiv g_{\alpha}-g_{\alpha}^{\text {eq }}=\frac{\tau_{g}}{\tau} f_{\alpha}^{\text {neq }} .
$$

In principle, the statistical information about the displacement of particles on the lattice is carried by $f_{\alpha}$. This implies in particular that local probability budgets should be developed with $f_{\alpha}$ rather than with $g_{\alpha}$. Importantly, one can notice that $f_{\alpha}$ does not depend on the lattice resolution, whereas the non-equilibrium part of $g_{\alpha}$ does obviously depend on $\Delta t$ through the lattice parameter $\tau_{g}$. Despite these differences, the confusion between $f_{\alpha}$ and $g_{\alpha}$ has often been made in previous works (as specified later), leading to a depreciating of the accuracy of the method (since it amounts to ignore a term of order $\Delta t$ in Eq. (4)).

The integration of Eq. (5) is usually organized in a two-step "collide-and-stream" algorithm, in which the collision step accounts for the re-distribution of the particles among the different directions of propagation,

$$
g_{\alpha}^{\text {out }}(\mathbf{x}, t)=g_{\alpha}(\mathbf{x}, t)-\frac{1}{\tau_{g}^{*}}\left(g_{\alpha}(\mathbf{x}, t)-g_{\alpha}^{\mathrm{eq}}(\mathbf{x}, t)\right) \text { with } \tau_{g}^{*} \equiv \frac{\tau_{g}}{\Delta t},
$$

whereas the streaming step transports the particles (according to their post-collision velocity) to their neighboring lattice nodes,

$$
g_{\alpha}\left(\mathbf{x}+\mathbf{c}_{\alpha} \Delta t, t+\Delta t\right)=g_{\alpha}^{\text {out }}(\mathbf{x}, t)
$$

This algorithm is explicit, easy to implement and straightforward to parallelize, and thus achieves a very high performance on common computing systems [12]. However, these advantages are inherently connected with the use of a regular lattice with a constant spacing. This is in contrast with conventional discretization methods in which the grid may be profitably refined locally to gain in accuracy while minimizing the computational efforts (as 


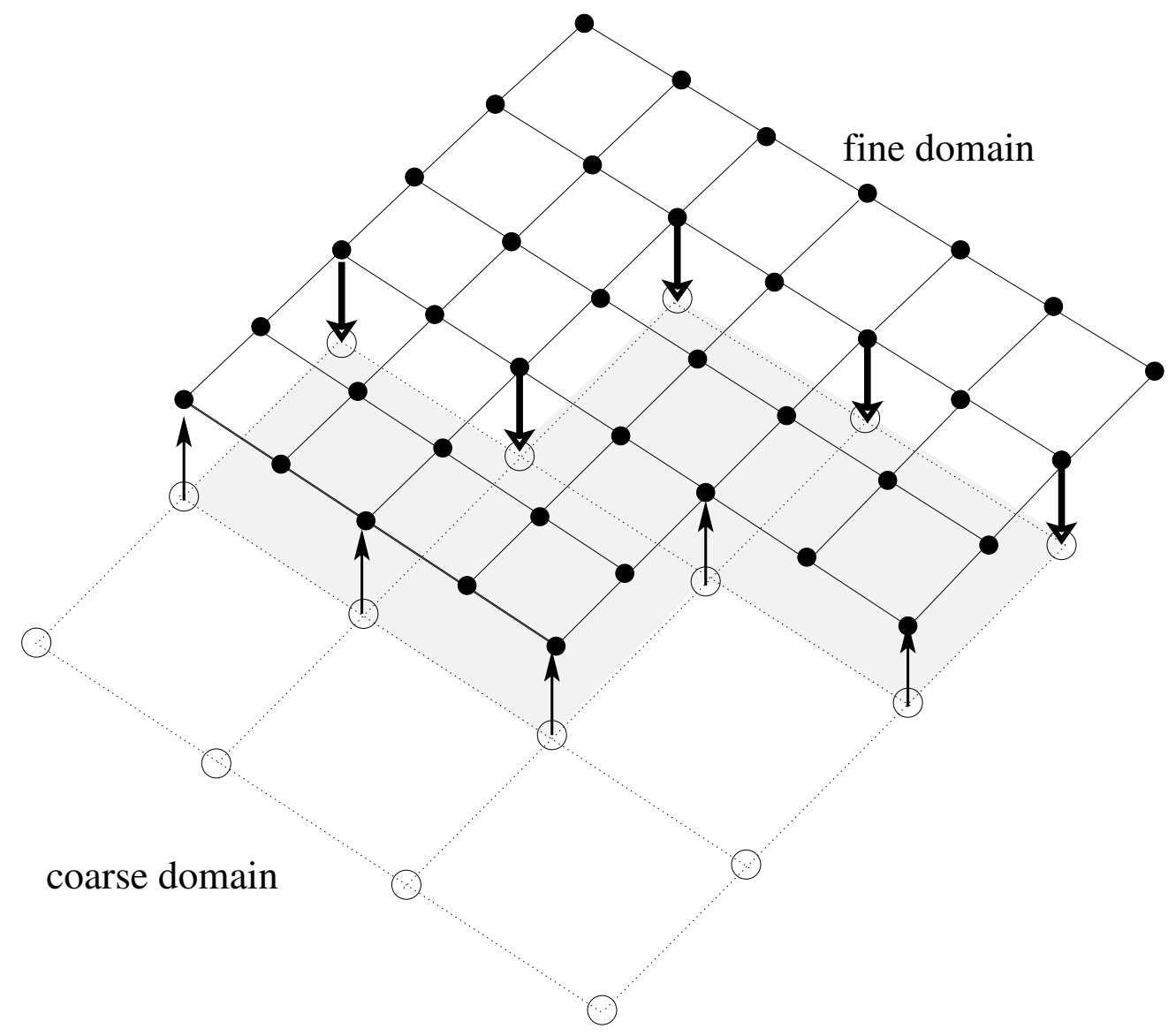

FIG. 3: In the transition zone (in grey) between two domains of different resolutions, it is required to map the probability distributions from the fine interior nodes to the coarse boundary nodes (downwards arrow), and vice-versa from the coarse interior nodes to the fine boundary nodes (upwards arrow). The probability distributions at intermediate fine boundary nodes can be reconstructed by 3rd-order interpolation in space and time (at odd timesteps) [13].

recalled in the introduction). Therefore, in order for the LB method to remain competitive compared to other discretization methods, it should be adapted to handle composite multi-resolution grids, especially in the context of turbulent flows.

A number of variants have been proposed to port the LB method to non-uniform grids (in a cell-vertex representation). First proposals rely on the combination of the LB method with finite-volume or finite-difference techniques on irregular grids [14, 15]. Nonetheless, such combination can only accomodate relative smooth varations of the flow due to numerical instabilities triggered by the deformation of the grid. Another solution is the so-called "locally embedded uniform grids" technique. Namely, the embedding (within the root grid) 
of domains in which the local connectivity is unchanged but the grid spacing is different, typically multiplied or divided by a power of two (see Fig. 1). Here, the advantage is to place domains with a smaller spacing in flow regions which require more accuracy, and coarser domains in less demanding regions. Within each domain, the lattice is uniform and the computational performance of the method is preserved. However, some inconsistency in the connectivity of boundary nodes requires to correctly map the probability distributions at the transition between two domains (Fig. 3).

Lin and Lai [16] suggested on the basis of probabilistic arguments that the distributions $g_{\alpha}$ should be directly transfered between two domains without any transformation. To our understanding, this method makes the confusion between $f_{\alpha}$ and $g_{\alpha}$. Indeed, while the (genuine) distributions $f_{\alpha}$ may be transferable to some degree between two domains, the (lattice-based) distributions $g_{\alpha}$ surely cannot since they depend on the local grid resolution. As a result, this technique only ensures the continuity of the mass density and fluid momentum (because $\rho$ and $\rho \mathbf{u}$ can be recovered equivalently from $g_{\alpha}^{\text {eq }}$ and $f_{\alpha}^{\text {eq }}$ ) but introduces some spurious error in the continuity of the viscous stress (per unit mass), which depends explicitly on the non-equilibrium part of the distributions [11]. Precisely,

$$
\sigma_{i j}^{\text {visc. }} \propto \frac{\tau}{\tau_{g}} \sum_{\alpha} c_{\alpha i} c_{\alpha j} g_{\alpha}^{\text {neq }}=\sum_{\alpha} c_{\alpha i} c_{\alpha j}\left(\frac{\tau}{\tau_{g}} g_{\alpha}^{\text {neq }}\right)=\sum_{\alpha} c_{\alpha i} c_{\alpha j} f_{\alpha}^{\text {neq }} .
$$

The continuity of the viscous stress requires to account for the variation of the factor $\tau / \tau_{g}$ at resolution changes. This issue has been addressed by Filippova and Hänel [17] who suggested to rescale adequately $g_{\alpha}^{\text {neq }}$ in the mapping. Interestingly, Filippova and Hänel's method eventually amounts to transfering directly $f_{\alpha}$ according to Eq. (9). Later on, Dupuis and Chopard [18] have introduced a method that resembles Filippova and Hänel's method but is simpler, and avoids a singularity when $\tau_{g}=\Delta t / 2$. Concretely, they have suggested a rescaling of $g_{\alpha}^{\text {neq }}$ before the collision step, whereas Filippova and Hänel had considered a rescaling after the collision step. Finally, Lagrava et al. [13] have very recently proposed to improve futher Filippova and Hänel's method by applying a local average (restricted to the first neighboring nodes) to $g_{\alpha}^{\text {neq }}$ on the fine grid before performing the mapping to the coarse grid. The authors claim that this procedure is expected to filter out unphysical grid-scale excitations and "guarantee the stability of the numerical scheme at high Reynods numbers". Two-dimensional test calculations at moderate Reynolds numbers have indeed established that the stability was improved, nonetheless, fully developed turbulent flows (in 
three dimensions) have not been examined in their study.

In section III, the mapping conditions at the transition between two domains of different resolutions will be reconsidered on a more general basis. This description will allow us to recast previous modeling (reported above) in a unified framework, and extend it to address the LES of turbulent flows.

\section{MAPPING OF PROBABILITY DISTRIBUTIONS AT RESOLUTION CHANGES}

\section{A. General assumptions}

As detailed before, the continuous discrete-velocity Boltzmann equation is amenable to space-and-time dicretization to eventually yield the lattice Boltzmann equation. Conceptually, the LB solution is therefore expected to represent the locally coarse-grained (at a scale comparable to the lattice spacing) solution of the original problem. According to Eq. (1), this coarse-grained (or smoothed) solution, $\overline{f_{\alpha}}$, a priori satisfies

$$
\frac{\mathrm{D} \overline{f_{\alpha}}}{\mathrm{D} t} \equiv \frac{\partial \overline{f_{\alpha}}}{\partial t}+c_{\alpha i} \cdot \frac{\partial \overline{f_{\alpha}}}{\partial x_{i}}=-\overline{\left(\frac{f_{\alpha}^{\text {neq }}}{\tau}\right)} \approx-\frac{{\overline{f_{\alpha}}}^{\text {neq }}}{\tau}
$$

where the last approximation on the "smoothed collision term" will hold provided that the lattice spacing is sufficiently fine to resolve the finest details of the (exact) solution. In that situation, $\overline{f_{\alpha}}$ is therefore solution of the original discrete-velocity Boltzmann equation, and is thus expected to give an accurate representation of the exact solution: $\overline{f_{\alpha}} \approx f_{\alpha}$. This is the traditional context of a Direct Numerical Simulation (DNS). Importantly, Eq. (10) does not depend explicitly on the lattice resolution, which implies that the probability distributions $\overline{f_{\alpha}}$ may be directly exchanged at resolution changes. This is indeed the underlying assumption of the Filippova and Hänel's method, which thus happens to be justified in the limit of sufficiently fine lattices. However, if the lattice spacing is not sufficiently fine, unresolved subgrid-scale variations of the (exact) solution must be taken into account in the

approximation of the "smoothed collision term", and consequently $-\overline{\left(f_{\alpha}^{\text {neq }} / \tau\right)} \neq-\overline{f_{\alpha}}{ }^{\text {neq }} / \tau$. This is the general context of a Large-Eddy Simulation (LES).

When dealing with turbulent flows, a common thread is to assume that subgrid-scale effects may be taken into account by an additional (subgrid-scale) viscosity $\bar{\nu}_{\text {sgs }}$ in the 
viscous stress [19]. Within the LB approach, this heuristic assumption amounts to correct the relaxation time so that $[20]$

$$
\overline{\left(\frac{f_{\alpha}^{\text {neq }}}{\tau}\right)} \approx-\frac{{\overline{f_{\alpha}}}_{\tau+\bar{\tau}_{\text {sgs }}}^{\text {neq }}}{\text { with }} \quad \bar{\tau}_{\mathrm{sgs}} \equiv \frac{\bar{\nu}_{\mathrm{sgs}}}{c_{\mathrm{s}}^{2}} .
$$

In that situation, the LB solution a priori satisfies

$$
\frac{\mathrm{D} \overline{f_{\alpha}}}{\mathrm{D} t} \approx-\frac{{\overline{f_{\alpha}}}^{\text {neq }}}{\bar{\tau}}
$$

where the redefined relaxation time $\bar{\tau}(\mathbf{x}, t) \equiv \tau+\bar{\tau}_{\text {sgs }}(\mathbf{x}, t)$ is now a flow quantity that is bound to the local grid spacing through the subgrid-scale viscosity. Note that Eq. (11) can only be justified in the weakly compressible limit [21]. Also, it is worth mentioning that an alternative estimation of the "smoothed collision term" relying on the Approximate Deconvolution Model [22] has been recently introduced by Malaspinas and Sagaut [23]. This latter offers a more systematic estimation but is a priori heavier to handle numerically. In this study, we follow the common approach, which is simpler and computationally efficient.

Eq. (12) is fulfilled in each domain separately since $\bar{\tau}$ depends explictly on the local grid spacing. At a boundary node $\mathbf{x}_{b}$ between two domains (of different resolutions), the respective collision terms are expected to match according to

$$
\begin{aligned}
& -\frac{{\overline{f_{\alpha}}}^{\mathrm{c}, \text { neq }}}{\bar{\tau}^{\mathrm{c}}}\left(\mathbf{x}_{b}, t\right)=\left(\mathcal{R} *-\frac{{\overline{f_{\alpha}}}^{\mathrm{f}, \text { neq }}}{\bar{\tau}^{\mathrm{f}}}\right)\left(\mathbf{x}_{b}, t\right) \\
& \text { and }-\frac{{\overline{f_{\alpha}}}^{\mathrm{f}, \text { neq }}}{\bar{\tau}^{\mathrm{f}}}\left(\mathbf{x}_{b}, t\right)=\left(\mathcal{P} *-\frac{{\overline{f_{\alpha}}}^{\mathrm{c} \text {,neq }}}{\bar{\tau}^{\mathrm{c}}}\right)\left(\mathbf{x}_{b}, t\right) \text {, }
\end{aligned}
$$

where the convolution operators $\mathcal{R}$ and $\mathcal{P}$ are introduced formally to account for the rescaling of the collision term to the appropriate grid resolution. These operators are denoted by $\mathcal{R}$ and $\mathcal{P}$ in analogy with the restriction and prolongation operators used in multigrid or Adaptive Mesh Refinement techniques [24]. From now on, the superscripts ${ }^{\mathrm{f}}$ and $^{\mathrm{c}}$ refer respectively to the fine and coarse resolution. Also, the overbar symbol (used to indicate that the flow quantity is bound to the local grid spacing) is abandoned to simplify the notations.

According to Eq. (9), the previous matching conditions account for the discontinuity of the rate-of-strain tensor $\mathrm{S}_{i j} \propto \sigma_{i j}^{\text {visc. }} / \tau$ associated with the enlargement or refinement of the grid spacing: $\mathrm{S}_{i j}^{\mathrm{c}}\left(\mathbf{x}_{b}, t\right)=\left(\mathcal{R} * \mathrm{~S}_{i j}^{\mathrm{f}}\right)\left(\mathbf{x}_{b}, t\right)$ and $\mathrm{S}_{i j}^{\mathrm{f}}\left(\mathbf{x}_{b}, t\right)=\left(\mathcal{P} * \mathrm{~S}_{i j}^{\mathrm{c}}\right)\left(\mathbf{x}_{b}, t\right)$. Besides, the continuity of the mass and fluid momentum is ensured by imposing the matching of the 
equilibrium part of the probability distributions, i.e.

$$
f_{\alpha}^{\mathrm{f}, \mathrm{eq}}\left(\mathbf{x}_{b}, t\right)=f_{\alpha}^{\mathrm{c}, \mathrm{eq}}\left(\mathbf{x}_{b}, t\right)
$$

Eqs. (13) and (14) eventually define the full mapping of probability distributions at the transition between two domains (see Fig. 3). In terms of the lattice-based probability distributions, this mapping reformulates as

$$
\begin{aligned}
& g_{\alpha}^{\mathrm{f}, \text { eq }}\left(\mathbf{x}_{b}, t\right)=g_{\alpha}^{\mathrm{c}, \text { eq }}\left(\mathbf{x}_{b}, t\right) \\
& g_{\alpha}{ }^{\mathrm{c} \text { neq }}\left(\mathbf{x}_{b}, t\right)=\tau_{g}^{\mathrm{c}}\left(\mathbf{x}_{b}, t\right) \cdot\left(\mathcal{R} * \frac{g_{\alpha}^{\mathrm{f}, \text { neq }}}{\tau_{g}^{\mathrm{f}}}\right)\left(\mathbf{x}_{b}, t\right) \\
& g_{\alpha}{ }^{\mathrm{f}, \text { neq }}\left(\mathbf{x}_{b}, t\right)=\tau_{g}^{\mathrm{f}}\left(\mathbf{x}_{b}, t\right) \cdot\left(\mathcal{P} * \frac{g_{\alpha}^{\mathrm{c}, \text { neq }}}{\tau_{g}^{\mathrm{c}}}\right)\left(\mathbf{x}_{b}, t\right) .
\end{aligned}
$$

Importantly, this mapping has the merit of conserving the mass because the condition $\sum_{\alpha} g_{\alpha}^{\text {neq }}(\mathbf{x}, t)=0$ is preserved. To be fully determined, an additional relation between $\tau_{g}^{\mathrm{f}}\left(\mathbf{x}_{b}, t\right)$ and $\tau_{g}^{\mathrm{c}}\left(\mathbf{x}_{b}, t\right)$ is also required; for instance, $\tau_{g}^{\mathrm{c}}\left(\mathbf{x}_{b}, t\right)$ is unkown in the rhs of Eq. (15b) and needs to be expressed as a function of $\tau_{g}^{\mathrm{f}}\left(\mathbf{x}_{b}, t\right)$. Because these lattice relaxation times are expected to account for (possible) subgrid-scale turbulent dynamics, this relation should rely on some turbulence modeling. This is now detailed.

\section{B. Accounting for subgrid-scale turbulent dynamics}

In general, the lattice relaxation time $\tau_{g}$ expresses (in lattice units denoted by the symbol *) as

$$
\tau_{g}^{*}(\mathbf{x}, t)=\frac{1}{2}+3\left(\nu^{*}+\nu_{\mathrm{s} g s}^{*}(\mathbf{x}, t)\right)
$$

with the normalized viscosity defined as $\nu^{*} \equiv \nu / 3 c_{\mathrm{s}}^{2} \Delta t$. By construction, the spacing and the timestep of the lattice are related by $\Delta x / \Delta t=\sqrt{3} c_{\mathrm{s}}$. Since $\Delta x^{\mathrm{c}}=2 \Delta x^{\mathrm{f}}$, this implies that $\Delta t^{\mathrm{c}}=2 \Delta t^{\mathrm{f}}$ and $\nu^{* \mathrm{f}}=2 \nu^{* \mathrm{c}}$. From Eq. (16), one therefore gets

$$
2 \tau_{g}^{* \mathrm{c}}\left(\mathbf{x}_{b}, t\right)=\tau_{g}^{* \mathrm{f}}\left(\mathbf{x}_{b}, t\right)+\frac{1}{2}+3\left(2 \nu_{\mathrm{sgs}}^{* \mathrm{c}}\left(\mathbf{x}_{b}, t\right)-\nu_{\mathrm{sgs}}^{* \mathrm{f}}\left(\mathbf{x}_{b}, t\right)\right)
$$

Generally speaking, a kinematic viscosity is a diffusion coefficient that may be viewed as the product of a characteristic length scale and a characteristic velocity (at that scale) [19]. Concerning the subgrid-scale viscosity, these two characteristic quantities should be identified with the local grid spacing, $\Delta x$, and the typical velocity difference at this grid 
scale, $\delta u(\Delta x)$. According to Kolmogorov's theory, one can establish that $\delta u(\Delta x) \sim(\Delta x)^{1 / 3}$ [25]. The change of grid resolution (by a factor two) then leads to

$$
\nu_{\mathrm{sgs}}^{* \mathrm{c}}\left(\mathbf{x}_{b}, t\right)=2^{1 / 3} \nu_{\mathrm{sgs}}^{* \mathrm{f}}\left(\mathbf{x}_{b}, t\right)
$$

and finally

$$
2 \tau_{g}^{* \mathrm{c}}\left(\mathbf{x}_{b}, t\right)=\tau_{g}^{* \mathrm{f}}\left(\mathbf{x}_{b}, t\right)+\frac{1}{2}+3\left(2^{4 / 3}-1\right) \nu_{\mathrm{sgs}}^{* \mathrm{f}}\left(\mathbf{x}_{b}, t\right) \quad \text { with } \quad \nu_{\mathrm{sgs}}^{* \mathrm{f}} \equiv \frac{\nu_{\mathrm{sgs}}^{\mathrm{f}}}{3 c_{s}^{2} \Delta t} .
$$

The contribution related to the subgrid-scale dynamics is explicitly taken into account by the last term in the rhs of Eq. (19). This contribution automatically vanishes with the subgrid-scale viscosity if there is no subgrid-scale turbulence. In that case, the standard relation between the lattice relaxation times is recovered [17]. It should be noted that Eq. (19) is general and does not depend on the specific modeling of the subgrid-scale viscosity, e.g. the classical Smagorinsky model [2]. It also ensures that $\tau_{g}^{*}$ remains greater than one half - a condition for the stability of the LB scheme — provided that the subgrid-scale viscosity is kept positive [43].

\section{Restriction and prolongation operators}

The restriction and prolongation operators involved in the mapping need to be specified. The restriction accounts for the rescaling of the collision term from a fine grid to a coarse grid resolution. To be compliant with the geometry of the D3Q19 lattice and to remain simple, it is here proposed to achieve this coarse-graining by a full-weighted volumic average accounting for the 19 neighbors (including the node itself). For the collision term $C_{\alpha}^{\mathrm{f}}(\mathbf{x}, t)$ resolved on the fine lattice, this transformation reads

$$
\left(\mathcal{R} * C_{\alpha}^{\mathrm{f}}\right)(\mathbf{x}, t)=\frac{1}{7} C_{\alpha}^{\mathrm{f}}(\mathbf{x}, t)+\frac{1}{14} \sum_{\beta=1}^{6} C_{\alpha}^{\mathrm{f}}\left(\mathbf{x}+\mathbf{c}_{\beta} \Delta t^{\mathrm{f}}, t\right)+\frac{1}{28} \sum_{\beta=7}^{18} C_{\alpha}^{\mathrm{f}}\left(\mathbf{x}+\mathbf{c}_{\beta} \Delta t^{\mathrm{f}}, t\right),
$$

where the three contributions correspond respectively to the "center", "faces" and "edges" neighboring nodes (see Fig. 2). The volumic weights are respectively 1, 1/2 and 1/4, and a normalization factor is used to set the sum of all coefficients equal to one.

More generally, a Gaussian box-filter with half-width $\sigma \approx \Delta x^{\mathrm{c}} / 2$ could be considered for the restriction operation. In that case, one obtains that $\mathcal{R} \approx \mathbb{I}+\left(\sigma^{2} / 2\right) \Delta$ at leading order. 
By discretizing the Laplacian operator $\Delta$ on a stencil corresponding to the D3Q19 molecule [26], one gets

$$
\Delta C_{\alpha}^{\mathrm{f}}(\mathbf{x}, t) \approx \frac{1}{6\left(\Delta x^{\mathrm{f}}\right)^{2}}\left(-24 C_{\alpha}^{\mathrm{f}}(\mathbf{x}, t)+2 \sum_{\beta=1}^{6} C_{\alpha}^{\mathrm{f}}\left(\mathbf{x}+\mathbf{c}_{\beta} \Delta t^{\mathrm{f}}, t\right)+\sum_{\beta=7}^{18} C_{\alpha}^{\mathrm{f}}\left(\mathbf{x}+\mathbf{c}_{\beta} \Delta t^{\mathrm{f}}, t\right)\right)
$$

and finally

$$
\begin{aligned}
\left(\mathcal{R} * C_{\alpha}^{\mathrm{f}}\right)(\mathbf{x}, t) & =\left(1-2 \sigma^{* 2}\right) C_{\alpha}^{\mathrm{f}}(\mathbf{x}, t) \\
& +\frac{\sigma^{* 2}}{12}\left(2 \sum_{\beta=1}^{6} C_{\alpha}^{\mathrm{f}}\left(\mathbf{x}+\mathbf{c}_{\beta} \Delta t^{\mathrm{f}}, t\right)+\sum_{\beta=7}^{18} C_{\alpha}^{\mathrm{f}}\left(\mathbf{x}+\mathbf{c}_{\beta} \Delta t^{\mathrm{f}}, t\right)\right)
\end{aligned}
$$

with $\sigma^{*} \equiv \sigma / \Delta x^{\mathrm{f}}$ in lattice units. Eq. (21) yields a more general formulation of the restriction operator, in which $\sigma^{*}$ may be viewed as a free parameter (with $\sigma^{*}$ close to 1). Our proposal based on a (basic) volumic average corresponds to the specific value $\sigma^{*}=\sqrt{3 / 7} \simeq 0.7$. In practice, we have checked that the whole method was not sensitive to the precise value of $\sigma^{*}$ and that our (arbitrary) choice was satisfactory.

Concerning the prolongation from a coarse grid to a fine grid, a common practice is to simply copy the value at coinciding nodes and resort to interpolation at intermediate fine nodes [13]. As a matter of fact, this is a "low-level approximation" that does not explicitly account for the enrichment of turbulent fluctuations on the fine grid [27]. In a LES, this enrichment is a priori essential since turbulent excitations are expected to populate the whole resolution bandwidth. Nonetheless, we have disregarded this feature (as a first step) in our study and prolongation has been estimated by simple (3rd-order) interpolation. Test computations for a turbulent channel flow (reported in section IV) will show that this neglect does not significantly impact the results.

In principle, the prolongation is expected to "de-smooth" the collision term and recreate some grid-scale excitations on the fine grid. However, this operation is delicate and prone to numerical instability. Our attempts to approximate the prolongation operator by simply inverting the previous Gaussian box-filter: $\mathcal{P} \approx \mathbb{I}-\left(\sigma^{2} / 2\right) \Delta$, have resulted in strong perturbations of the solution. Finally, even if an improved procedure may be desirable in fine for the prolongation procedure, we believe that "enrichment" is already partially achieved in our coarse-to-fine mapping by accounting explicitly for turbulence in Eq. (19) between $\tau_{g}^{* \mathrm{c}}\left(\mathbf{x}_{b}, t\right)$ and $\tau_{g}^{* \mathrm{f}}\left(\mathbf{x}_{b}, t\right)$. 


\section{Unified framework}

Interestingly, if one excludes the restriction operation and disregards subgrid-scale effects, our proposal eventually reduces to the formula introduced by Filippova and Hänel [17] and revisited later by Dupuis and Chopard [18]. As previously argued, the domain of validity of these approaches is restricted to DNS on sufficiently fine grids (in order to validate Eq. (10)). If one retains the restriction operation but disregards subgrid-scale effects, our proposal resembles the method proposed recently by Lagrava et al. [13] by identifying the restriction with their filtering procedure. This latter has proved itself to improve the numerical stability of the LB scheme in presence of strong gradients but does not explicitly account for subgridscale effects. In this regard, it is therefore also restricted to DNS. Our proposal may be viewed as an attempt to conciliate these different aspects in order to suitably address the LES of turbulent flows.

\section{TESTS COMPUTATIONS OF A TURBULENT CHANNEL FLOW}

A turbulent channel flow has been used to test the capabilities of our multi-resolution LB scheme (Fig. 4). This academic flow configuration is of importance in engineeringflow problems and is commonly used as a prototypical case to investigate wall-bounded turbulence [28]. It is well documented in the literature and can benefit in particular from the comprehensive database built by Moser et. al [29].

Our LB simulations are refered to as LaBS (for Lattice Boltzmann solver) in the following. The scheme is based on the standard D3Q19 lattice and relies on the BGK approximation. The speed of sound is arbitrary and fixed to optimize the long-time convergence of the simulation while ensuring that the Mach number remains small, typically Ma $\approx 0.15$. The no-slip condition is enforced at the boundaries by using the method developed by Verschaeve and Muller [30], which is a generalization of the model introduced by Skordos [31] to offlattice boundaries. The strategy of this boundary treatment is to accurately interpolate or extrapolate macroscopic flow quantities at boundary nodes, and reconstruct the probability distributions from these quantities. In our simulation, the first fluid node is at a distance $0.5 \Delta x$ (half-spacing) from the wall. This method is consistent with the overall second-order accuracy (in space) of the LB scheme. A uniform body force is added to the dynamics in 


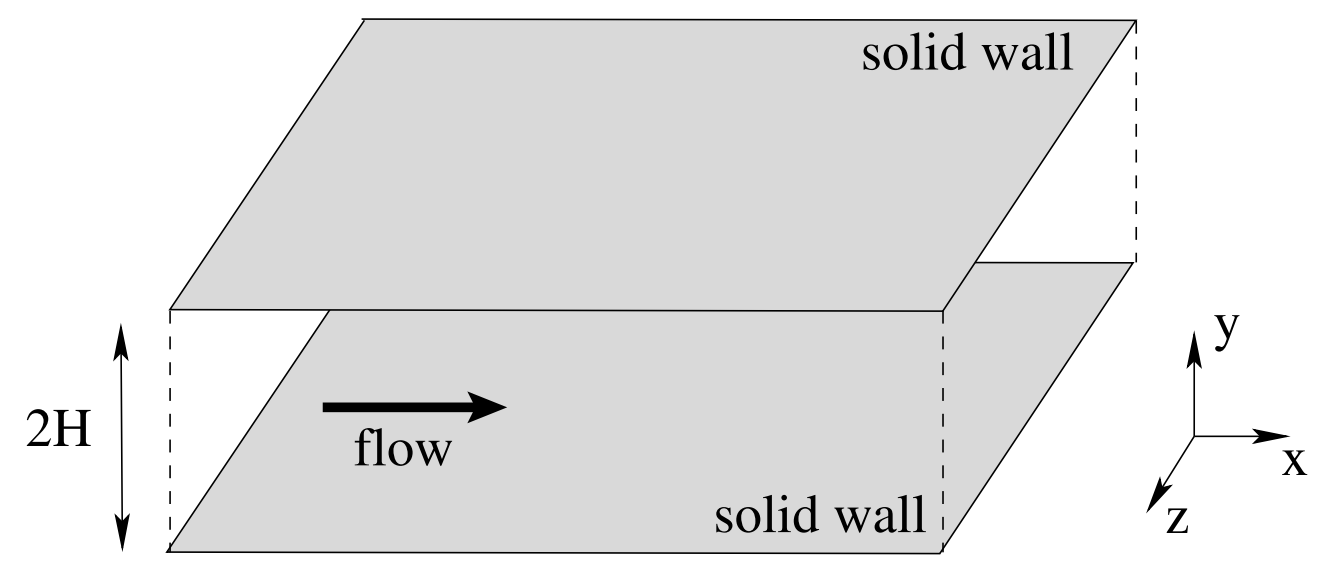

FIG. 4: Bi-periodic plane-channel flow. The flow is driven along the $x$-direction by a uniform body force (adjusted in time to reach an objective averaged flow rate). Periodic boundary conditions are used in the streamwise and spanwise directions. No-slip boundary conditions are considered at the wall.

the streamwise direction to drive the flow against the viscous forces. This force is adjusted dynamically to reach the target averaged flow rate across the channel, as detailed in [32]. In the LB scheme, this external body force is included in the collision step according to the procedure established by Guo et al. [33]. Finally, the subgrid-scale viscosity relies on the shear-improved Smagorinsky model (SISM) introduced by Lévêque et al. [34]. Namely,

$$
\nu_{\mathrm{sgs}}(\mathbf{x}, \mathbf{t})=\left(\mathrm{C}_{s} \Delta x\right)^{2}(|\mathrm{~S}(\mathbf{x}, t)|-|\widetilde{\mathrm{S}}(\mathbf{x}, t)|)
$$

where $\mathrm{C}_{s}$ is the Smagorinsky constant, $\Delta x$ is the local grid spacing and $\mathrm{S}(\mathbf{x}, t)$ is the resolved rate of strain. Here, the rate of strain cannot be easily computed from the distributions; it is better evaluated by differentiating the reconstructed velocity field. The correcting term to the Smagorinsky viscosity is the norm of the averaged rate of strain. The average (indicated by the tilde) is here meant in time and is estimated by using a recursive exponential filter, as detailed in [35].

The SISM accomodates the effects pertaining to the presence of a strong shear by reducing significantly the value of the Smagorinsky viscosity in that situation, e.g. near a solid boundary. Interestingly, this model does not call for any adjustable parameter besides the Smagorinsky constant $\mathrm{C}_{s} \approx 0.18$ that is fixed for all flows [19]. There is no need for $a d-h o c$ (Van Driest) damping function nor any kind of dynamic adjustment [2]. The simplicity and the manageability of the original Smagorinsky model are therefore preserved. The SISM has 


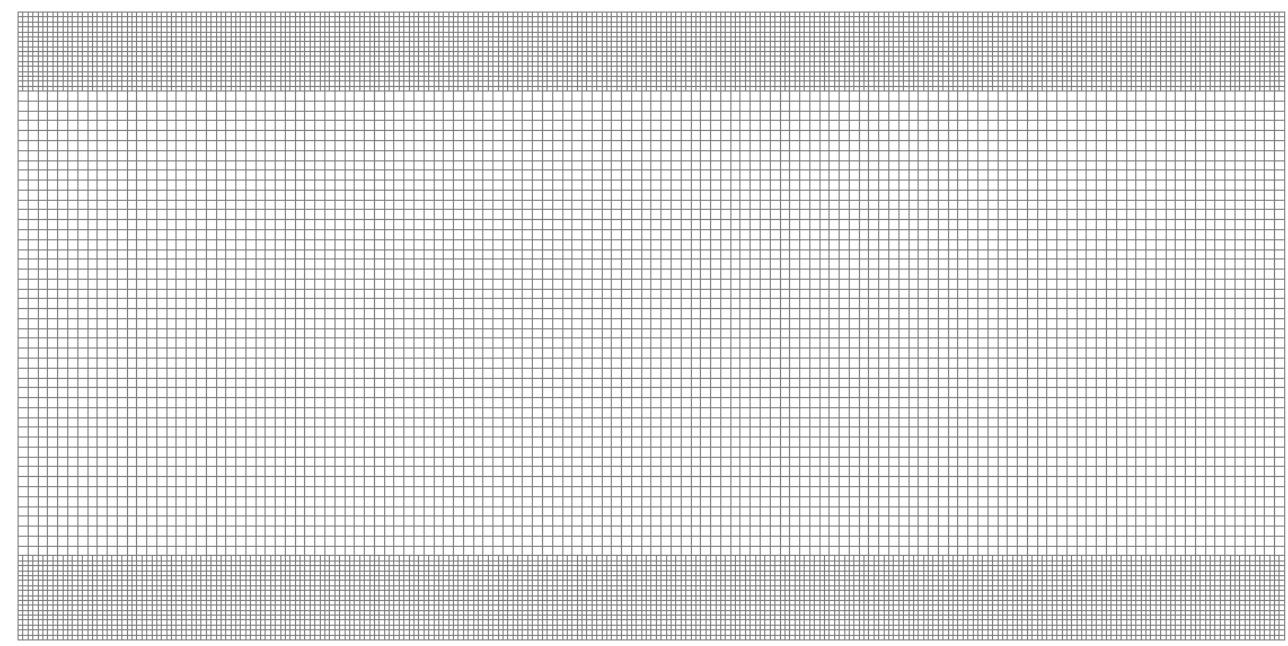

FIG. 5: Sketch of the grid of our test simulation at $\operatorname{Re}_{\tau}=180$. The grid spacing is $\Delta y^{+} \approx 2.7$ in the near-wall zones, whereas $\Delta y^{+} \approx 5.3$ in the bulk. The transition between the two domains occurs at $y^{+} \approx 40$.

already validated in various flow configurations [34-36].

In the following, the grid-scale velocity components are $U+u^{\prime}, v^{\prime}$ and $w^{\prime}$ along the streamwise, wall-normal and spanwise directions, respectively. $U(y)$ is the mean velocity, whereas $u^{\prime}(\mathbf{x}, t), v^{\prime}(\mathbf{x}, t)$ and $w^{\prime}(\mathbf{x}, t)$ are (instantaneous) turbulent velocities. The distance to the wall is $y$. The Reynolds number is defined as $\operatorname{Re}_{\tau}=u_{\tau} H / \nu$, where $u_{\tau}=\sqrt{\tau_{w} / \rho}$ is the skin-friction velocity and $\tau_{w}=\rho \nu|\mathrm{d} U(y) / \mathrm{d} y|_{\text {wall }}$ is the averaged shear stress at the wall. As usual, the distance to the wall and the velocity are normalized according to $y^{+}=y u_{\tau} / \nu$ and $u^{+}=u / u_{\tau}$ (wall units).

Two Reynolds numbers (both referenced in the database published by Moser et al. [29]) are considered. In a first simulation at $\operatorname{Re}_{\tau}=180$, the grid resolution is refined in the boundary zones to resolve the finest structures of the flow and perform a (quasi-)DNS. The grid resolution is much coarser in a LES at $\operatorname{Re}_{\tau}=395$.

\section{A. Quasi-DNS at $\operatorname{Re}_{\tau}=180$}

An important requirement of our scheme is that reference high-resolution results should be recovered in the limit of a sufficiently fine grid. This is the motivation of our first test simulation at $\operatorname{Re}_{\tau}=180$. A sketch of the grid is displayed in Fig. 5. The domain size 

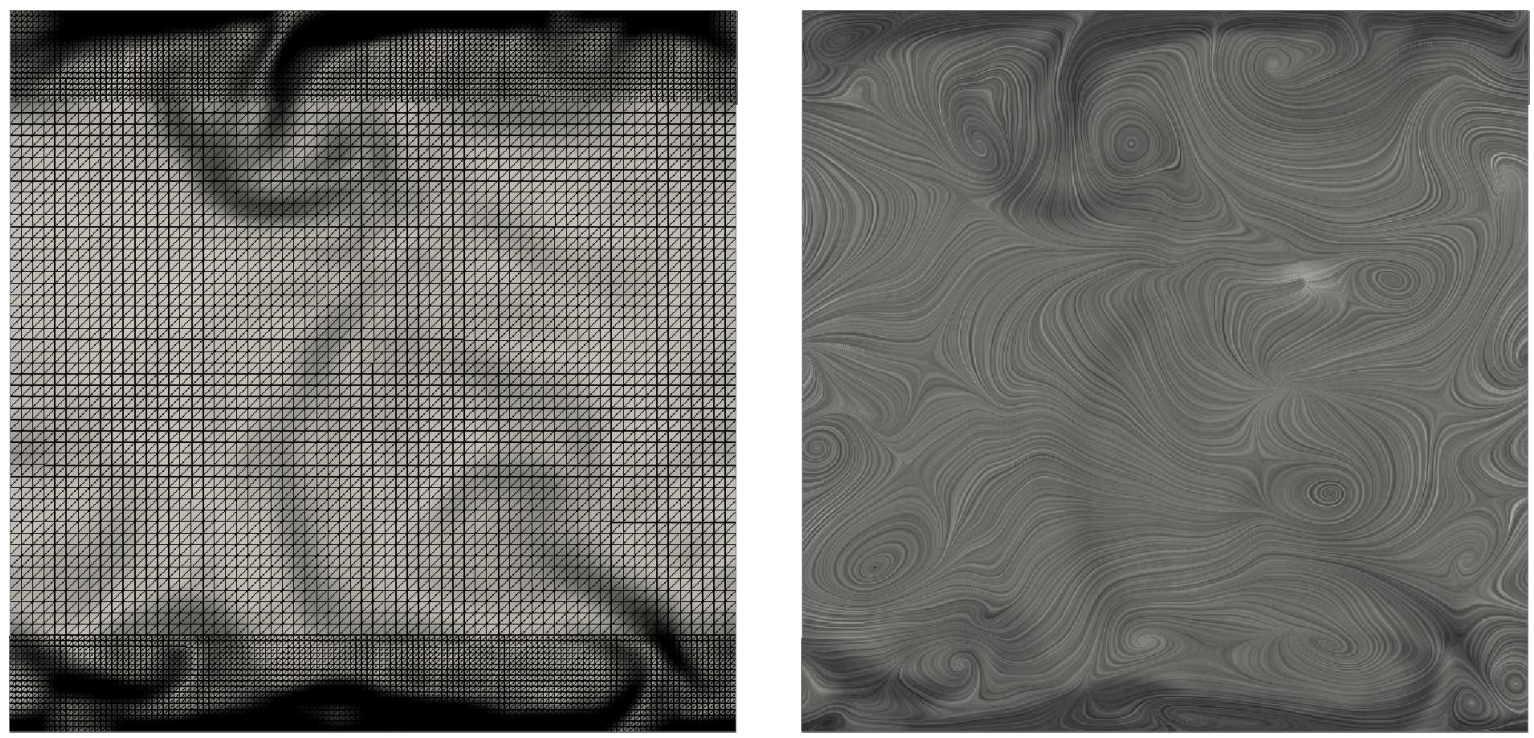

FIG. 6: Test simulation at $\operatorname{Re}_{\tau}=180$. Left: The magnitude of the streamwise component of the vorticity is displayed in greyscale in a plane normal to the mean flow (at an arbitrary time). Right: The corresponding streamlines of the projected velocity field (in the plane).

follows the minimum specifications for a periodic channel flow given by Jiménez and Moin [37]. The lattice is refined (by a factor two) in the viscous and transition layers, whereas a coarser resolution is used in the logarithmic layer. Accordingly, the transition between the two domains occurs at $y^{+} \approx 40$. The high-resolution Fourier-Chebyshev pseudo-spectral simulation performed by Moser et al. [29] is considered as reference. A comparison between the two grid configurations is summarized in Table I. The resolutions are relatively similar except in the vicinity of the walls, where $\Delta y^{+}$is significantly coarser in LaBS. Only 6 points are used in the range $0<y^{+}<10$, whereas 13 points are used in the pseudo-spectral simulation.

\begin{tabular}{|c|c|c|c|c|c|}
\hline simulation & domain size & grid size & $\Delta x^{+}$ & $\Delta y^{+}$(from wall to center) & $\Delta z^{+}$ \\
\hline Reference DNS & $4 \pi H \times 2 H \times \frac{4}{3} \pi H$ & $128 \times 129 \times 128$ & 17.7 & $0.05-4.4$ & 5.9 \\
\hline LaBS & $4 H \times 2 H \times 2 H$ & $128 \times 65 \times 64$ & $2.7,5.3$ & $(1.3) 2.7,5.3$, & $2.7,5.3$ \\
\hline
\end{tabular}

TABLE I: Grid configurations at $\operatorname{Re}_{\tau}=180$. In LaBS, the grid size is given in coarse-cell units. The grid cell is cubic: the resolution in the streamwise and spanwise direction is imposed by the wall-normal resolution. The first fluid node (near the wall) is at half-spacing, i.e. $y^{+} \approx 1.3$.

Qualitatively, the streamwise component of the vorticity (related to the presence of 

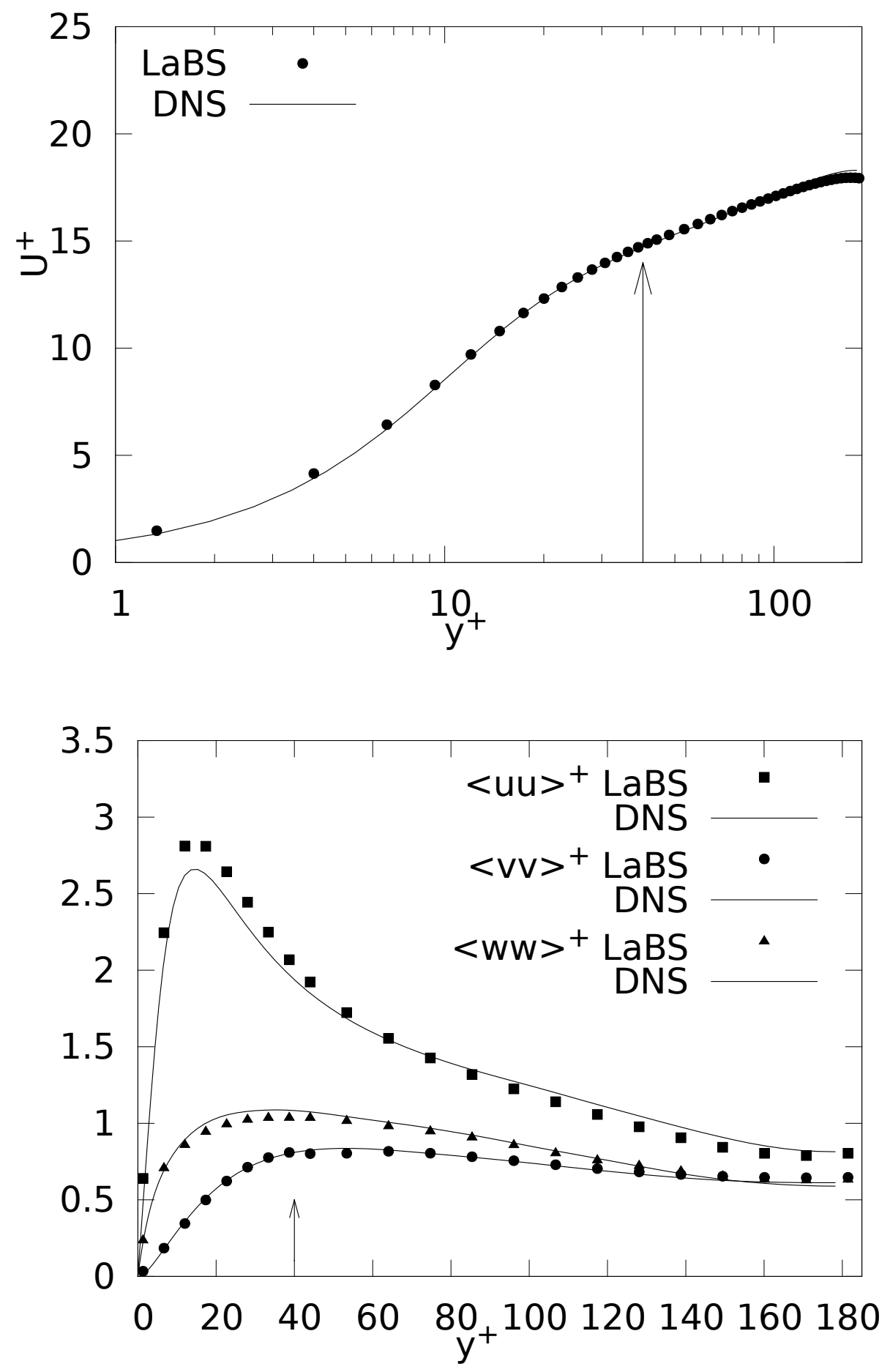

FIG. 7: Test simulation at $\operatorname{Re}_{\tau}=180$. Top: Mean velocity as a function of the distance to the wall (in wall units). Bottom: Mean-squared turbulent velocity fluctuations. The change of grid resolution in LaBS is indicated by the arrow. 


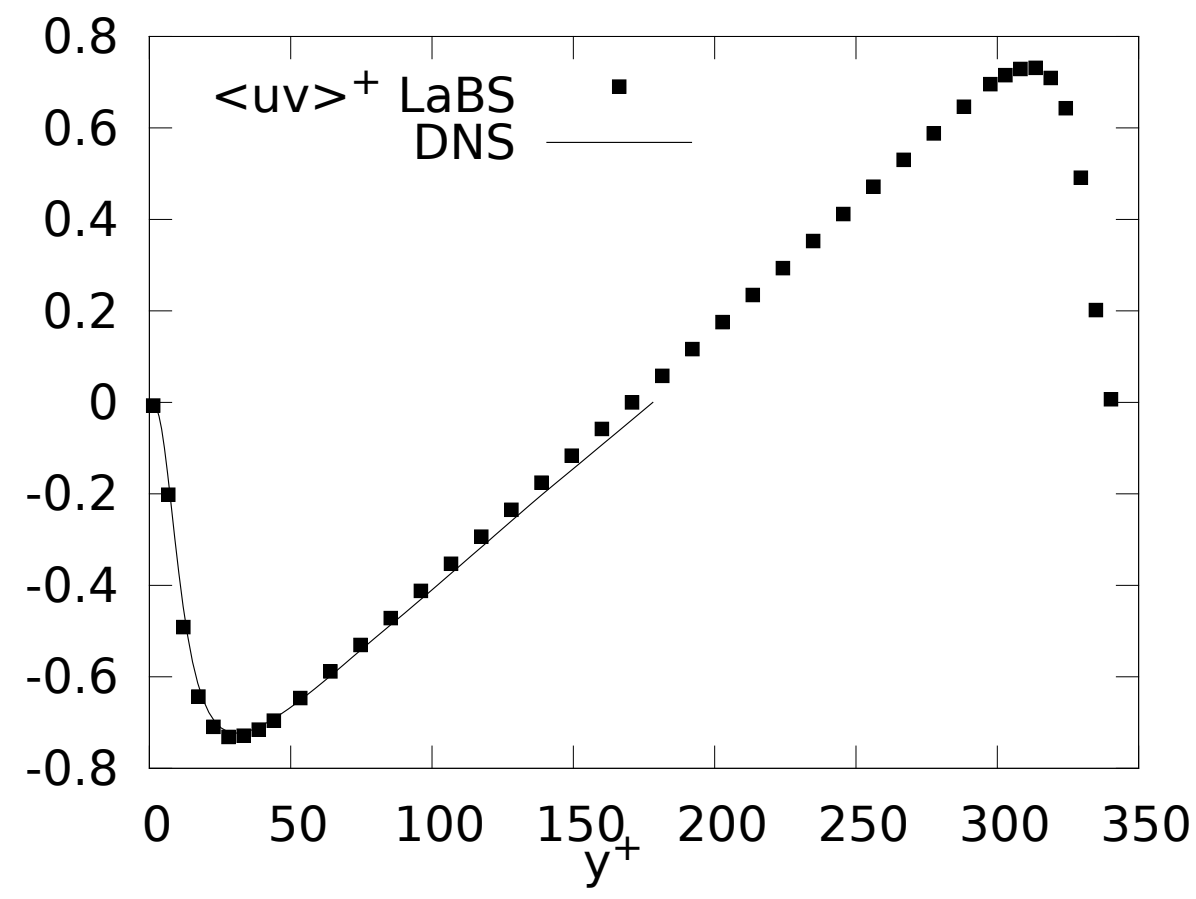

FIG. 8: The Reynolds stress at $\operatorname{Re}_{\tau}=180$.

streamwise vortices near the wall) seems to be suitably prolongated from the fine and the coarse domain (see Fig. 6). The streamlines of the projected velocity do not show any notable discontinuity. These observations are consistent with the physical conditions used to derive the mapping of probability distributions at resolution changes.

As previously argued, our scheme is here expected to reduce to Filippova and Hänel's method [17] with an additional restriction procedure in the fine-to-coarse mapping. Interestingly, we have verified that Filippova and Hänel's scheme alone was unstable (in the same grid configuration) and that the restriction operation was necessary to prevent the growth of spurious excitations. This observation is consistent with the results obtained by Lagrava et al. [13] at moderate Reynolds numbers (in two dimensions). It is also worth mentioning that several recent papers have reported stable multi-resolution simulations of turbulent flows (in a plane channel [38] or past a sphere [39]) resorting to the Filippova and Hänel's method without any restriction procedure. However, these simulations rely on the so-called MRT scheme [40] instead of the standard BGK scheme. In that case, a good behavior is recovered by using a Multi-Relaxation-Time modeling of the collision operator that is specifically "adjusted" to improve the stability of the scheme. In return the computational cost of the 


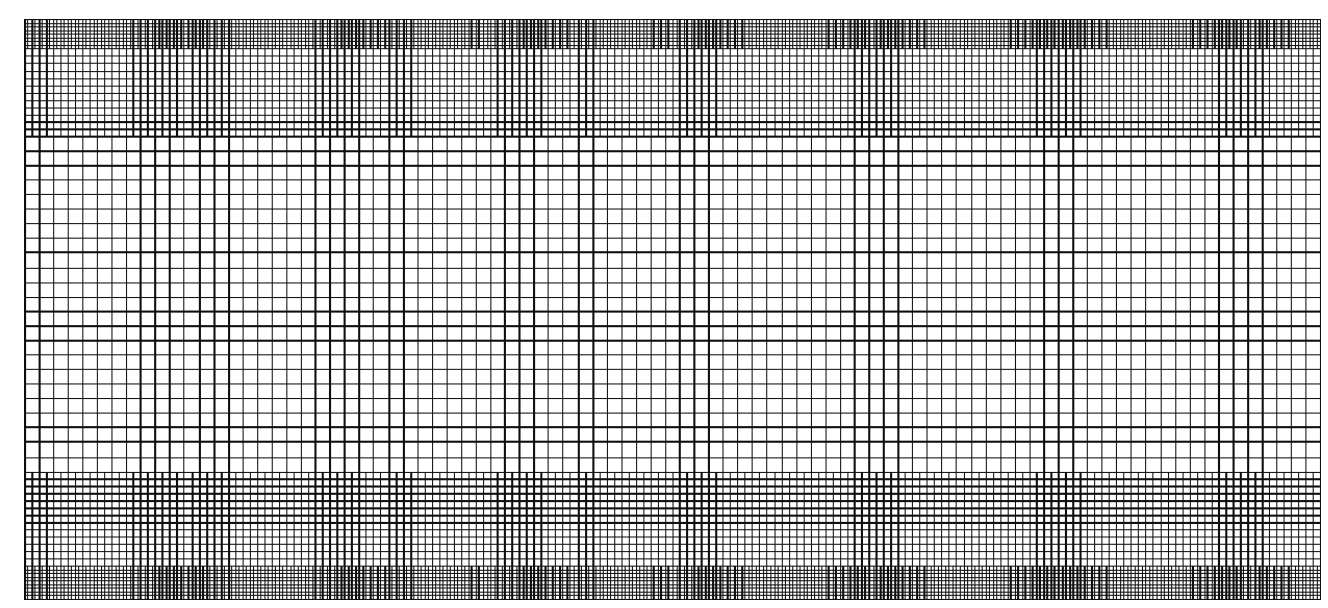

FIG. 9: Sketch of the grid at $\operatorname{Re}_{\tau}=395$. Three refinement levels are used. The lattice spacing is $\Delta y^{+} \approx 5$ near the walls and is coarsened successively by a factor two. The transitions are at $y^{+} \approx 40$ and $y^{+} \approx 150$ (in the logarithmic-layer).

\begin{tabular}{|c|c|c|c|c|c|}
\hline simulation & domain size & grid size & $\Delta x^{+}$ & $\Delta y^{+}$(from wall to center) & $\Delta z^{+}$ \\
\hline DNS & $2 \pi H \times 2 H \times \pi H$ & $256 \times 193 \times 192$ & 10 & $0.03-6.5$ & 6.5 \\
\hline LES-FV & $2 \pi H \times 2 H \times \pi H$ & $49 \times 89 \times 41$ & 52 & $0.5-24$ & 31 \\
\hline LaBS & $4.5 H \times 2 H \times 3 H$ & $89 \times 41 \times 59$ & $5,10,20$ & $(2.5) 5,10,20$, & $5,10,20$ \\
\hline
\end{tabular}

TABLE II: Grid configurations at $\operatorname{Re}_{\tau}=395$. In LES-FV, the grid spacing is stretched in the $y$-direction by a hyperbolic tangent function.

scheme is heavier. Our position is somehow opposite since we want to remain as simple as possible in the physical modeling of the collision operator but focus on the improvement of the mapping conditions.

The mean velocity and the turbulence intensities are displayed as a function of the wallnormal distance in Fig. 7. The various profiles compare very well with the reference DNS data. The change of resolution is hardly noticeable even in the turbulence intensity profiles. The Reynolds-stress profile is shown in Fig. 8 and the agreement is also very satisfactory.

\section{B. LES at $R e_{\tau}=395$}

In a second simulation at $\operatorname{Re}_{\tau}=395$, the grid resolution has been deliberately reduced so as to perform a LES. A sketch of the grid is displayed in Fig. 9. LaBS results are compared 

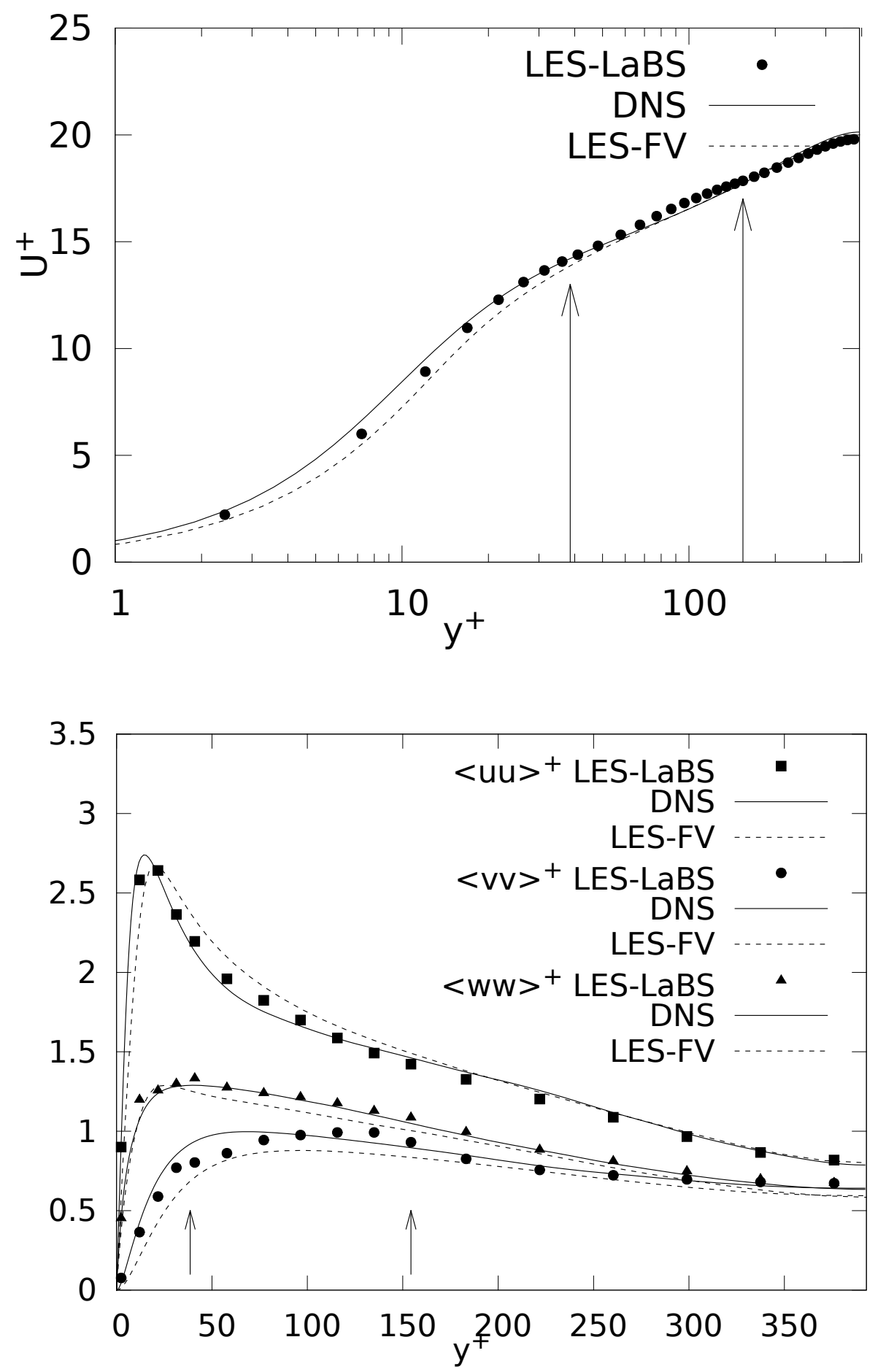

FIG. 10: Simulations at $R e_{\tau}=395$. Top: mean velocity as a function of the wall distance (in wall units). Bottom: mean-squared velocity fluctuations. LaBS results (symbols) are compared with high-resolution pseudo-spectral data (DNS) and results obtained from a LES (with the same subgrid-scale viscosity) based on finite-volume discretization (LES-FV). The two arrows indicate the change of resolution in LaBS. 


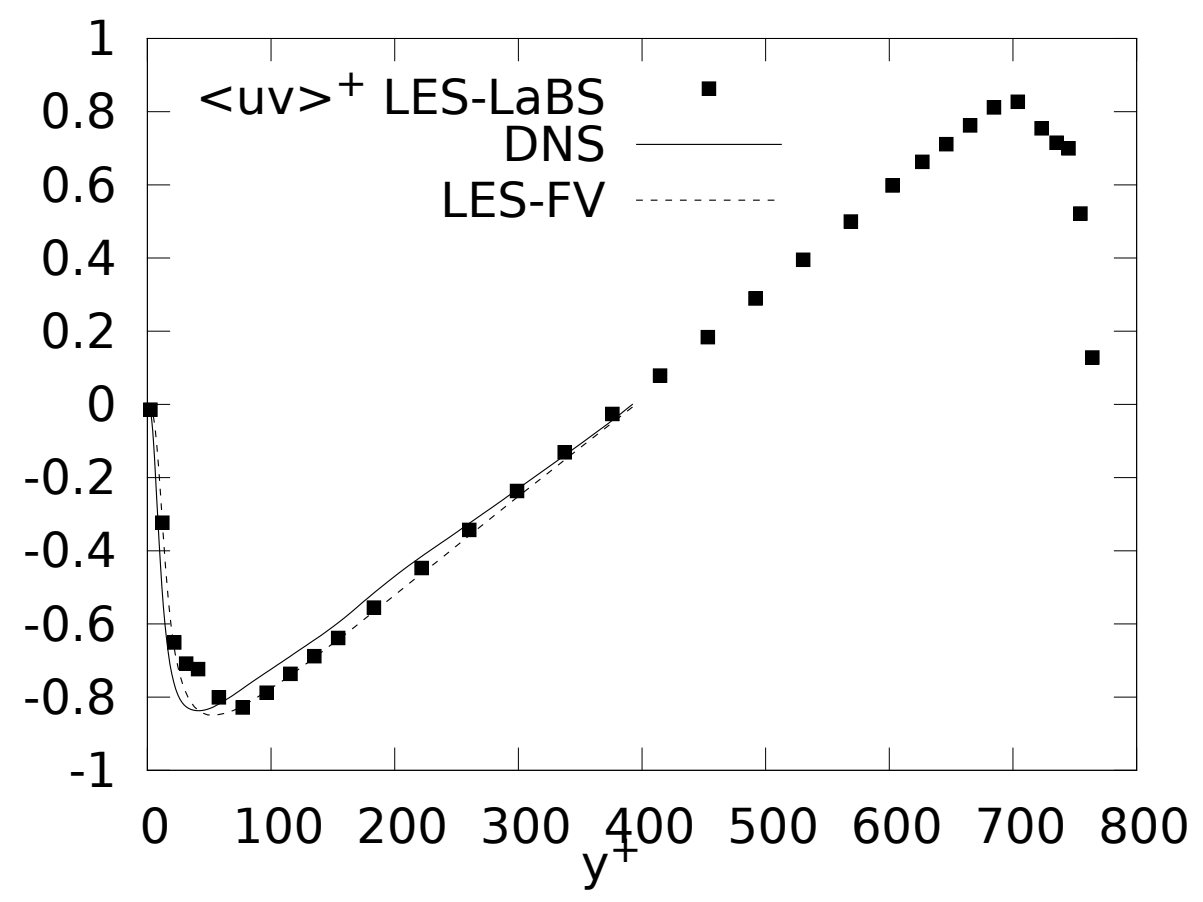

FIG. 11: The Reynolds stress at $R e_{\tau}=395$.

with both high-resolution pseudo-spectral data [29] and the results obtained from an alternative LES with the same subgrid-scale viscosity but relying on finite-volume discretization (LES-FV). In LES-FV, convective fluxes are interpolated with a four-point centered scheme (fourth-order accuracy on regular grid) and diffusive fluxes with a two-point centered scheme (second-order). The time stepping is driven by a five-step Runge-Kutta scheme. More details about this code may be found in [41]. Importantly, the resolutions are comparable except in the near-wall region where the grid resolution (in the wall-normal direction) is again much coarser in LaBS. The different grid configurations are summarized in Table II.

First of all, one must point out that the simulation remained stable. The mean-velocity and turbulent-intensity profiles are shown in Fig. 10. The reference velocity (used in the normalization) is here the objective friction velocity of the DNS $\left(u_{\tau}=0.59 \mathrm{~m} \cdot \mathrm{s}^{-1}\right)$ and not the individual friction velocity of each simulation. This setting allows us for a direct comparison between the different simulations. Moreover, it makes the nondimensional meancenterline velocity match the reference DNS value, which is consistent with the strategy of calibration of the extenal forcing. A good prediction of the overall mean-velocity profile is achieved as compared to the DNS data. The two LES yield similar results with LaBS being 
slightly closer to the DNS in the transition layer. This is remarkable in consideration of the relatively low resolution used in the near-wall region. Indeed, only 3 points are used in the range $0<y^{+}<10$ in LaBS, whereas 11 grid points are used in LES-FV (as standardly recommended [2]). This observation brings out the (intrinsic) capability of the LB scheme to handle strong shear layers. Concerning the turbulent-intensity profiles, they are very well captured for the streamwise and spanwise components. This is not so surprising since the resolution in these two directions is significantly finer than in LES-FV and almost comparable to the DNS resolution. Nevertheless, the two changes of resolution are very well handled. The overall profile of the wall-normal component is also well captured but a small depletion is observed at $y^{+} \gtrsim 40$ in the coarser domain near the first resolution jump. This depletion is also noticeable on the Reynolds stress in Fig. 11. The second change of resolution (in the logarithmic layer) is much better handled. The cause of this small depletion remains unclear to us. Our simplified prolongation procedure may be questioned at first. However, similar depletion was also observed by Quéméré et al. [27] by using an improved enrichment procedure in the context of finite-volume multi-domain/multi-resolution LES. Therefore, it seems to be inherent to the decrease of resolution.

\section{CONCLUSION}

In summary, this study addresses in a physically-sound framework the multi-resolution simulations of turbulent flows by the lattice Boltzmann method. Our approach allows us to conciliate previous works in a unified description, and extend them to suitably account for possible subgrid-scale turbulent dynamics in the context of LES. Importantly, the key Eq. (19) governing the mapping of lattice relaxation times (at resolution changes) is general and does not explicitly depend on the subgrid-scale viscosity model. Also, our study relies on the BGK approximation for the collision operator, but a similar approach could a priori be followed for any other approximation, e.g. the MRT approximation.

Rohde et al. [42] have recently advocated a cell-centered formulation (rather than the standard cell-vertex description) to address multi-resolution lattice Boltzmann simulations. In that case, the inconsistency in the connectivity of boundary nodes is alleviated, and our restriction and prolongation operations are naturally accounted by simply redistributing (merging or splitting) the populations of particles within embedded grid cells. In that 
context, our approach suggests (i) that such redistribution should be applied to the genuine probability distributions $f_{\alpha}$ instead of the lattice-based distributions $g_{\alpha}$ (see discussion in section II) and (ii) that Eq. (19) should be profitably integrated in the scheme to account for possible subgrid-scale turbulent dynamics.

Finally, we believe that the great amenability of the LB method to fluid dynamics computing, which is to a large extent preserved in the multi-resolution version, is expected to provide an asset for future applications to complex turbulent flows.

\section{Acknowledgements}

We acknowledge P. Lacabanne, B. Gaston, J.-P. Lahargue for their inputs in the development of the LaBS solver, and P. Sagaut for fruitful discussions about the 1 attice Boltzmann method and its application to the LES of turbulent flows. The authors would like to acknowledge the French Ministry of Industry (DGCIS) for its financial support of the LaBS project. Numerical simulations have been performed by using local HPC facilities at the Ens de Lyon (PSMN) supported by the grants CPER-CIRA (2007-2013) and FR-3403.

[1] M. Lesieur, Turbulence in Fluids (Kluwer, 1997).

[2] P. Sagaut, Large-Eddy Simulation for Incompressible Flows: An introduction (Springer, Berlin, 2001).

[3] G. R. McNamara and G. Zanetti, Phys. Rev. Lett. 61, 2332 (1988).

[4] C. Aidun and Clausen, Annu. Rev. Fluid Mech. 42, 439 (2010).

[5] P. Sagaut, Comput. Math. Appl. 59, 2194 (2010), ISSN 0898-1221.

[6] S. Chen and G. D. Doolen, Annu. Rev. Fluid Mech. 30, 329 (1998), ISSN 0066-4189.

[7] Y. H. Qian, D. d'Humières, and P. Lallemand, Europhys. Lett. 17, 479 (1992), ISSN 02955075 .

[8] S. Succi, The Lattice Boltzmann Equation for Fluid Dynamics and Beyond (Oxford Univesity, Oxford, 2001).

[9] P. L. Bhatnagar, E. P. Gross, and M. Krook, Phys. Rev. 94, 511 (1954).

[10] X. W. Shan, X. F. Yuan, and H. D. Chen, J. Fluid Mech. 550, 413 (2006), ISSN 0022-1120. 
[11] R. Benzi, S. Succi, and M. Vergassola, Phys. Rep. 222, 145 (1992), ISSN 0370-1573.

[12] M. Schoenherr, K. Kucher, M. Geier, M. Stiebler, S. Freudiger, and M. Krafczky, Comput. Math. Appl. 61, 3730 (2011).

[13] D. Lagrava, O. Malaspinas, J. Latt, and B. Chopard, J. Comp. Phys. 231(14), 4808 (2012).

[14] F. Nannelli and S. Succi, J. Stat. Phys. 68, 401 (1992), ISSN 0022-4715.

[15] R. W. Mei and W. Shyy, J. Comp 143, 426 (1998), ISSN 0021-9991.

[16] C. L. Lin and Y. G. Lai, Phys. Rev. E 62, 2219 (2000), ISSN 1063-651X.

[17] O. Filippova and D. Hanel, J. Comp. Phys. 147, 219 (1998), ISSN 0021-9991.

[18] A. Dupuis and B. Chopard, Phys. Rev E 67 (2003), ISSN 1063-651X.

[19] S. B. Pope, Turbulent Flows (Cambridge University Press, UK, 2000).

[20] H. Chen, S. Succi, and S. Orszag, Phys. Rev. E, Rap. Comm. 59, R2527 (1999).

[21] O. Malaspinas and P. Sagaut, J. Fluid Mech. 700, 514 (2012).

[22] S. Stolz and A. Adams, Phys. Fluids 11, 1699 (1999).

[23] O. Malaspinas and P. Sagaut, Phys. Fluids 23, 105103 (2011).

[24] M. J. Berger and P. Colella, J. Comp. Phys. 82, 64 (1989).

[25] U. Frisch, Turbulence: The Legacy of Kolmogorov (Cambridge University Press, UK, 1995).

[26] R. C. O’Reilly and J. M. Beck, Int. J. Numer. Meth. Engng 00, 1 (2006).

[27] P. Quéméré, P. Sagaut, and V. Couaillier, Int. J. Numer. Methods Fluids 36, 391 (2001).

[28] I. Marusic, B. J. McKeon, P. A. Monkewitz, H. M. Nagib, A. J. Smits, and K. R. Sreenivasan, Phys. Fluids 22, 065103 (2010).

[29] R. D. Moser, J. Kim, and N. N. Mansour, Phys. Fluids 11, 943 (1999).

[30] J. C. G. Verschaeve and B. Muller, J. Comp. Phys. 229, 6781 (2010), ISSN 0021-9991.

[31] P. A. Skordos, Phys. Rev. E 48, 4823 (1993), ISSN 1063-651X.

[32] E. Lenormand, P. Sagaut, and L. T. Phuoc, Int. J. Num. Meth. Fluids 32, 369 (2000).

[33] Z. L. Guo, C. G. Zheng, and B. C. Shi, Phys. Rev. E 65 (2002), ISSN 1063-651X.

[34] E. Lévêque, F. Toschi, L. Shao, and J. P. Bertoglio, J. Fluid Mech. 570, 491 (2007).

[35] A. Cahuzac, J. Boudet, P. Borgnat, and E. Lévêque, Phys. Fluids 22, 125104 (2010).

[36] F. Toschi, H. Kobayashi, U. Piomelli, and G. Iaccarino, in Proceedings of the Summer Programm CTR, Stanford (2006).

[37] J. Jiménez and P. Moin, J. Fluid Mech. 225, 213 (1991).

[38] K. N. Premnath, M. J. Pattison, and S. Banerjee, Physica A: Statistical Mechanics and its 
Applications 388, 2640 (2009), ISSN 0378-4371, URL http://www.sciencedirect.com/ science/article/B6TVG-4VTCM6K-1/2/bdb9a7ebde71de9753ba8db2d2251986.

[39] M. Stiebler, M. Krafczyk, S. Freudiger, and M. Geier, Comput. Math. Appl. 61, 3475 (2011).

[40] D. d'Humieres, I. Ginzburg, M. Krafczyk, P. Lallemand, and L. S. Luo, Philosophical Transactions of the Royal Society of London Series A-Mathematical Physical and Engineering Sciences 360, 437 (2002), ISSN 1364-503X.

[41] J. Boudet, J. Caro, L. Shao, and E. Lévêque, J. Therm. Sci. 16, 328 (2007).

[42] M. Rohde, D. Kandhai, D. J. J., and H. E. A. van den Akker, Int. J. Numer. Meth. Fluids 51, 439 (2006).

[43] A detailed calculation shows that the stability condition $\tau_{g}^{*}>1 / 2$ is actually preserved provided that $\nu_{\mathrm{sgs}}>-\nu / 2^{4 / 3}$. 